\title{
B-03
}

\section{EFECTOS DE LOS REGADÍOS DEL DELTA DEL EBRO SOBRE LA CALIDAD DE SUS MASAS DE AGUA ASOCIADAS}

\author{
Martínez Beltrán, J.
}

Centro de Estudios Hidrográficos del CEDEX, Paseo Bajo de la Virgen del Puerto 3, 28005 Madrid, julian.martinezbeltran@cedex.es

\section{Resumen}

El delta, situado al final del valle inferior del Ebro, tiene un área de aproximadamente 32.533 ha. El agua derivada en el azud de Xerta a los canales de la derecha e izquierda del Ebro riega 22.123 ha en el delta, de las que unas 21.400 ha son arrozales.

El clima es de tipo mediterráneo con carácter marítimo-fluvial, con una precipitación y una evapotranspiración anual media respectivamente de $526 \mathrm{~mm}$ y $1.016 \mathrm{~mm}$. El delta es la llanura de inundación del Ebro sobre un medio marino. Los suelos son arenosos en las planicies litorales y aluviales de profundidad y textura variables en el resto. Tiene asociadas varias masas de agua superficial y un acuífero superficial y otro profundo, ambos salinos.

Además de los dos canales, la red de riego consta de acequias primarias, secundarias y terciarias que conducen por gravedad el agua hasta las tomas de las fincas. Una red abierta de desagües, primarios y secundarios y de circunvalación de las lagunas, conduce los retornos de riego y el exceso de agua de lluvia a estaciones de bombeo y compuertas basculantes que los evacuan al mar, a las dos bahías y al propio Ebro y su brazo de Migjorn.

El balance de sales, estimado a partir del de agua, está equilibrado en el conjunto de la campaña de riego. La salinidad de las tierras regadas está controlada mediante el riego y drenaje de los arrozales. La cuña de agua salina de la desembocadura del Ebro apenas aporta sales a las tierras adyacentes del Ebro.

La dotación media anual de un arrozal (unos $23.300 \mathrm{~m}^{3} / \mathrm{ha}$ ) es necesaria para el consumo del arroz y para controlar la salinidad del agua de los arrozales. Aproximadamente la mitad del agua de riego aplicada se convierte en agua de drenaje superficial.

El aporte de agua de drenaje con sales y nitratos al Ebro a las lagunas interiores y al acuífero superficial no es relevante. La masa de sales procedente de las tierras regadas va a las dos bahías y al mar abierto. El arroz consume una porción muy significativa de los nitratos aplicados con el agua de riego. Sin arroz la masa de nitratos que llegaría al mar sería mayor que la aportada actualmente.

\section{Introducción}

La agricultura de regadío tiene un efecto socioeconómico importante e incluso impactos ambientales favorables, pero detrae agua de una masa superficial o subterránea y evacua agua de drenaje a otras masas. El CEDEX ha estudiado los efectos del regadío en la detracción de agua y sobre la calidad del agua de las masas asociadas a una zona regable. En los últimos 5 años, por encomienda de la Dirección General del Agua (DGA), del Ministerio de Agricultura, Alimentación y Medio Ambiente, mediante un estudio metodológico aplicado a tres zonas regables de España, una de ellas la zona regada en el delta por el Canal de la Derecha del Ebro. Posteriormente, por encargo de la Confederación Hidrográfica del Ebro (CHE), ha estudiado en todo el delta la salinidad de suelos y aguas porque allí es un problema permanente.

Esos estudios han caracterizado los regadíos del delta para posteriormente analizar los efectos del riego y drenaje sobre las masas de agua asociadas, en lo que concierne a 
salinidad y nitratos, y han propuesto opciones para asegurar la sostenibilidad actual de su agricultura de regadío. A partir de balances de agua, los estudios estimaron la masa de sales evacuada por 4 cuencas de drenaje del delta a las bahías, durante los años hidrológicos del 2011-2012 al 2013-2014 y parte del 2014-2015. También la masa de nitratos evacuada en el hemidelta derecho en los años hidrológicos 2011-2012 y 2012-2013. Distinguen los balances tres períodos del año hidrológico: las campañas de riego (abriloctubre) y de medidas ambientales (octubre-enero); y febrero-abril sin aportes de agua de canal. Esas cuencas son las que tienen datos de caudal de drenaje $(Q)$, nitratos $\left(\mathrm{NO}_{3}\right)$ y conductividad eléctrica $(C E)$ de la Red de Indicadores Ambientales del Delta del Ebro (RIADE) de la CHE. Este artículo sintetiza los resultados de esos dos estudios.

\section{Características climáticas y edáficas del delta del Ebro}

El delta está situado al final del valle inferior del Ebro, en la provincia de Tarragona. Tiene una superficie de aproximadamente 32.533 ha: 20.165 ha en el hemidelta derecho y 12.368 ha en el izquierdo.

El clima es de tipo mediterráneo condicionado por su carácter marítimo-fluvial y por la orientación del valle inferior del Ebro. Las zonas costeras del delta tienen unas temperaturas mínimas algo más altas que en la parte alta junto al río y unas máximas algo menores. También la humedad relativa es más alta en la costa que en el interior. En Amposta, la precipitación media anual durante el período 1992-2013 fue $526 \mathrm{~mm}$, con cierta variabilidad interanual (322 mm en 2009 y $691 \mathrm{~mm}$ en 1995). Junio y julio, y en menor medida agosto, son los meses menos lluviosos. Las precipitaciones más intensas se dan en otoño, con 125 $\mathrm{mm}$ en un día para un periodo de retorno de 10 años (IRYDA, 1982). Los vientos de componente NW y $\mathrm{N}$ alcanzan una velocidad en la cabecera del delta mayor que en sus zonas costeras. Los de levante ocasionan temporales que elevan el nivel del mar hasta $1 \mathrm{~m}$. La evapotranspiración de referencia $\left(E T_{0}\right)$ anual, media de tres estaciones, fue $1.016 \mathrm{~mm}$ durante el período 2012-2104.

Las tierras del delta están situadas en la llanura de inundación del Ebro formada sobre un medio marino. Las llanuras deltaicas comienzan en ambos bancos del río y terminan en depresiones en torno a lagunas interiores. Están diseccionadas por dos antiguos cauces que partían del Ebro, uno por la derecha y otro por la izquierda. Próximas a las líneas de costa hay planicies litorales, claramente diferenciadas de la formación fluvial. Tramos bajos de varios abanicos aluviales y estrechas franjas de origen coluvial forman el límite con las tierras altas adyacentes. El espesor de los depósitos fluviales decrece paulatinamente en dirección al mar, a medida que las arenas de origen marino van siendo más superficiales. La arena marina tienen un espesor de hasta unos $10 \mathrm{~m}$ junto a las costas. La cota sobre el nivel del mar varía entre 3 y $2 \mathrm{~m}$ en los bancos del río, de 2 a $1 \mathrm{~m}$ en las llanuras y menos de $1 \mathrm{~m}$ en las depresiones y planicies litorales.

Los suelos de los bancos del Ebro son aluviales, profundos y estratificados, de textura franca pero sus subsuelos son poco permeables y la arena no aparece a menos de $2 \mathrm{~m}$ de profundidad. Los de los de los antiguos cauces son profundos y de textura fina. Los de las llanuras deltaicas son de textura fina con subsuelos de arena y arenas francas a profundidad variable, desde más de $1 \mathrm{~m}$ en las partes más altas hasta menos de 0,5 $\mathrm{m}$ en las más bajas. Los de las depresiones son de textura fina superficialmente con arena marina a aproximadamente $1 \mathrm{~m}$ y los de las planicies litorales son uniformemente arenosos. Hay suelos con horizontes de turba debidos al hidromorfismo asociado a manantiales de agua subterránea (ullales).

\section{Masas de agua superficial}

El Ebro y su brazo de Migjorn, lagunas interiores (La Encañizada y La Tancada en el hemidelta derecho, La Estrella en el izquierdo y Los Calaixos en la isla de Buda son las mayores), las bahías (El Fangar y Los Alfaques) y el mar abierto son masas de agua superficial asociadas al delta y receptoras de agua de drenaje (Figura 1). 


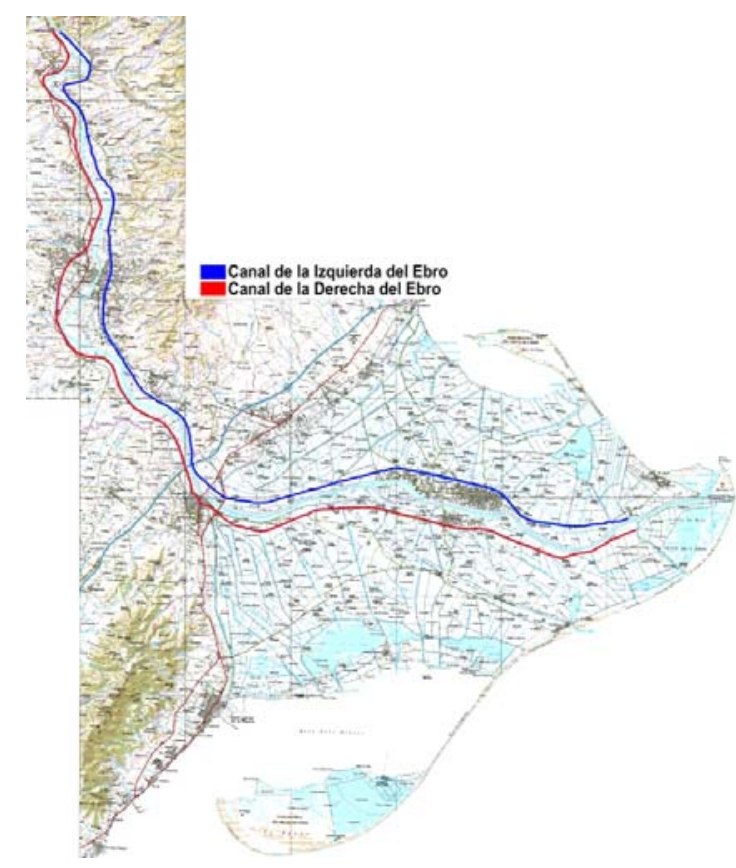

Figura 1. Canales de la derecha y de la izquierda del Ebro y sus zonas dominadas respectivas. Masas de agua superficial asociadas al delta (DGA/CEDEX, 2013)

El Ebro a su vez aporta el agua de riego, que tiene una concentración media de solidos disueltos totales (SDT) de $0,68 \mathrm{~g} / \mathrm{L}$ y un $\mathrm{pH}$ de 8 . Los aniones predominantes son sulfatos, bicarbonatos y cloruros; los cationes calcio y sodio y en menor medida magnesio. La CE media mensual varía desde $0,76 \mathrm{dS} / \mathrm{m}$ en mayo a $1,26 \mathrm{dS} / \mathrm{m}$ en octubre y progresivamente disminuye en otoño-invierno. La media en los últimos 12 años fue 0,98 dS/m. En el tramo final del Ebro la CHE (2010) determinó en 2009 y 2010 cambios de la CE del agua desde $3 \mathrm{dS} / \mathrm{m}$ a menos de $2-2,5 \mathrm{~m}$ a $57 \mathrm{dS} / \mathrm{m}$ a mayor profundidad, con una oscilación anual de $\pm 1,5 \mathrm{~m}$ en función del caudal y de la elevación del nivel del mar.

La salinidad del agua de las bahías varía a lo largo del año hidrológico. La CE del agua del estrato superior de la bahía Los Alfaques fue disminuyendo en julio de 2013 de 27 a $17 \mathrm{dS} / \mathrm{m}$, manteniéndose en agosto-septiembre entre 10 y $20 \mathrm{dS} / \mathrm{m}$, con la del estrato inferior en el intervalo de 35-45 dS/m. Al comienzo del año 2013-2014 la CE del agua del estrato superior era $53 \mathrm{dS} / \mathrm{m}$ y la del inferior $57 \mathrm{dS} / \mathrm{m}$, manteniéndose así durante el período sin riego de 2014, excepto en episodios con lluvias, durante los cuales bajó hasta 35-40 $\mathrm{dS} / \mathrm{m}$ y a $45-50 \mathrm{dS} / \mathrm{m}$ respectivamente. En la bahía de El Fangar también es ligeramente más salina la del estrato inferior que en el superior. Durante la campaña de riego de 2014 descendió ligeramente en mayo hasta 40-45 dS/m en el estrato superior y $45-50 \mathrm{dS} / \mathrm{m}$ en el inferior, para subir en ambos a 45-50 dS/m en julio. También aumentó en otoño, en el estrato inferior especialmente, con valores comprendidos entre 55 y $65 \mathrm{dS} / \mathrm{m}$.

En las campañas de riego de 2012 a 2014, la CE del agua de La Encañizada varió entre 30 y $35 \mathrm{dS} / \mathrm{m}$, pero en dos años (2012 y 2013) bajó en agosto hasta unos $15 \mathrm{dS} / \mathrm{m}$ de media. En septiembre y en ausencia de lluvias (2013 y 2014) subió a 50-55 dS/m, similar al del agua de Los Alfaques en esa época. Precipitaciones de otoño ocasionan descensos significativos, como ocurrió en noviembre y diciembre de 2014 (5-10 dS/m). Durante períodos sin lluvias de 2013 y 2014 la CE del agua se mantuvo entre 45 y 50 dS/m, pero durante cortos episodios de lluvias bajó hasta 30-35 dS/m.

\section{Masas de agua subterránea}

Hay dos acuíferos, uno superficial y otro profundo. El primero es detrítico y se extiende desde los bancos del Ebro hasta las líneas de costa. Está constituido por 
sedimentos aluviales sobre arena marina. En el hemidelta derecho limita con el acuífero carbonatado adyacente y en el izquierdo con los acuíferos superficiales de los abanicos aluviales. Tiene un espesor variable entre unos $10 \mathrm{~m}$ en los bancos y en las líneas de costa, y unos $5 \mathrm{~m}$ en las depresiones. Es libre excepto en los bancos y en las depresiones donde está semiconfinado por sedimentos de textura fina de menor permeabilidad que los de textura gruesa subyacentes. El límite inferior de este acuífero es un acuitardo, de espesor creciente desde el borde oeste del delta al mar, constituido por arcillas y limos saturados de agua salina en un medio reductor muy poco permeable.

El nivel freático está en la superficie del terreno por el riego de los arrozales y por la inundación de los campos con fines ambientales. Sin lluvias significativas de febrero a abril, desciende hasta casi $1 \mathrm{~m}$. Mientras se riega, el agua más superficial del acuífero es poco salina $(C E<5 \mathrm{dS} / \mathrm{m})$ y flota por su menor densidad sobre un agua salobre de entre 5 y 20 $\mathrm{dS} / \mathrm{m}$. Por debajo, tiene una de CE de 50-60 dS/m similar a la de mar. En las depresiones las aguas más profundas son salmueras de más de $80 \mathrm{dS} / \mathrm{m}$. Sin aportaciones a los campos queda el agua salobre en la parte superior del acuífero (DGA/CEDEX, 2013).

El plano de isohipsas (INC/Hydrotechnic, 1966) muestra flujo de los bancos a las depresiones de ambos hemideltas a través de las llanuras deltaicas, con un gradiente hidráulico del 0,2 al 1 por mil y también de los bancos al Ebro. Puede haber ocasionalmente gradiente del río a las tierras adyacentes durante crecidas, pero este flujo no es significativo debido a la baja permeabilidad del acuífero en los bancos, con valores de transmisividad hidráulica de 0,12 a 2,7 $\mathrm{m}^{2} / \mathrm{d}$ (CHE/IGME, 2014). Sin bancos, hay flujo del río al acuífero con un gradiente de 0,5 a 0,7 por mil. En las llanuras litorales hay flujo directo al mar o a las dos bahías con un gradiente de 0,2-0,5 por mil.

Por debajo del acuitardo existe un acuífero profundo confinado de espesor variable no conectado hidráulicamente con el superior. En el borde occidental del hemidelta derecho sí existe conexión con el acuífero carbonatado de la sierra de Montsiá, originando ullales cuya agua tiene una CE entre 1,6 y 2,2 dS/m (DGA/CEDEX, 2013).

\section{Redes de riego y de drenaje}

El agua del Ebro derivada en el azud de Xerta al canal de la derecha riega 12.715 ha en el delta y la derivada al canal de la izquierda 9.408 ha. Ambos canales riegan antes el valle inferior. En el delta discurren por la parte más alta de ambos bancos del río dominando las tierras cultivadas por lo que el riego es por gravedad. La Figura 1 muestra el trazado de los dos canales y de las acequias principales que de ellos derivan. Los dos canales son operados y mantenidos respectivamente por la Comunidad General de Regantes del Canal de la Derecha del Ebro (CGRCDE) desde 1970 y por la Comunidad de Regantes-Sindicato Agrícola del Ebro (CR-SAE) desde 1967. Ambas tienen concesiones anuales de agua de la $\mathrm{CHE}$, con caudales máximos de riego de unos $23 \mathrm{~m}^{3} / \mathrm{s}$ y $20 \mathrm{~m}^{3} / \mathrm{s}$ respectivamente.

El sistema de riego consta de una red de acequias primarias, derivadas de los dos canales, secundarias y terciarias que distribuyen el agua con un caudal continuo nominal $\left(q_{i}\right)$ de $1,84 \mathrm{ls}^{-1} \mathrm{ha}^{-1}$ en el hemidelta derecho y de $1,9 \mathrm{ls}^{-1} \mathrm{ha}^{-1}$ en el izquierdo. Las comunidades Ilenan los canales a mitad de abril y reducen progresivamente el caudal hasta alcanzar entre el 75 y el 80 por ciento del nominal al terminar la campaña de riego. Los regantes aplican el agua a los arrozales a partir de tomas de las acequias de finca. Solamente, de mayo a julio, interrumpen el riego para tratamientos y fertilización del arroz durante dos o tres episodios con un total de unos diez días. La dotación media anual para el riego de las variedades más cultivadas de arroz de ciclo largo es de unos $23.300 \mathrm{~m}^{3} / \mathrm{ha}$ (DGA/CEDEX, 2013).

La red de desagües, primarios y secundarios, es de zanjas abiertas con talud protegido por vegetación natural. Los primarios van a favor de la pendiente natural desde las tierras más altas hasta las estructuras de evacuación del agua de drenaje. Tienen capacidad para almacenar y conducir el exceso de agua de lluvia en 4 días durante las labores de recolección en septiembre-octubre, con una escorrentía de $31,2 \mathrm{~mm} / \mathrm{d}$ equivalente a un caudal de drenaje $\left(q_{d r}\right)$ máximo $3,61 \mathrm{Is}^{-1} \mathrm{ha}^{-1}$. Los desagües de circunvalación de las lagunas 
están dotados de compuertas que generalmente están cerradas, pero se abren cuando el nivel de agua en esas lagunas está por debajo del de los desagües.

Con objeto de evacuar el exceso de agua de lluvia y de riego de los arrozales, los regantes refinan los bancales, nivelados a cero, para eliminar cualquier depresión y evacuan el agua superficial al desagüe de último orden a través de una arqueta y una tubería de forma que no se erosione su talud. Sistemas de drenaje subterráneo, con drenes laterales y colectores de tubería, solamente hay en dos grandes fincas del hemidelta derecho. El caudal de drenaje subterráneo es elevado desde cubetas de bombeo a un emisario que desagua por gravedad.

El exceso de agua de lluvia se evacúa mediante 9 estaciones de bombeo con tornillos de Arquímedes y de 7 casetas con bombas de aspiración vertical, todas ellas situadas a lo largo de las líneas de costa con excepción de una estación del hemidelta izquierdo que está situada junto al Ebro. También durante la campaña de riego para rebajar el nivel del agua en la red de desagües, con objeto de facilitar el drenaje superficial en momentos críticos, como son los de tratamientos, fertilización y preparación del terreno para la recolección del arroz. Los retornos de riego, que originan menores caudales, se evacuan principalmente por gravedad a través de compuertas basculantes. Las tierras situadas entre los dos canales principales y el Ebro drenan por gravedad al río a través de bocanas. El agua del final de los primarios llega a las estaciones de evacuación mediante canales de aproximación.

En el hemidelta derecho hay 4 bombas de realimentación que pueden elevar el agua de drenaje y verterla en la red de riego. Estas bombas entran en operación si la CE del agua de drenaje es menor de $2 \mathrm{dS} / \mathrm{m}$, de forma que la del agua resultante tenga de 1,5 a 1,6 $\mathrm{dS} / \mathrm{m}$ de $C E$.

\section{Agricultura de regadío}

El arroz es el cultivo principal del delta por ser la única opción en la mayor parte de sus tierras, que carecen de drenaje natural y solamente disponen de sistemas de drenaje superficial. Durante 2014 los agricultores cultivaron arroz en aproximadamente 12.600 ha en el hemidelta derecho y 8.800 ha en el izquierdo, unas 21.400 ha en total (97 por ciento del área total cultivada). Cultivan variedades de arroz de ciclo largo (180 días) y menos de ciclo corto (140 días). La Tabla 1 describe la duración de las fases de cultivo de las de ciclo largo.

\begin{tabular}{|l|l|c|}
\hline Fases & Fecha & $\begin{array}{c}\text { Número } \\
\text { de días }\end{array}$ \\
\hline Siembra, germinación y fase de plántula & 15 abril a 17 mayo & 32 \\
\hline Crecimiento y ahijado & 18 mayo a 24 julio & 68 \\
\hline Espigado y formación del grano & 25 julio a 25 agosto & 32 \\
\hline Maduración hasta la recolección & 26 agosto a 12 octubre & 48 \\
\hline
\end{tabular}

Tabla 1.Duración de las fases de cultivo de variedades de arroz de ciclo largo (CGRCDE)

Las máximas necesidades de agua las tiene el arroz al final de la fase de crecimiento y comienzo de la de espigado, con un consumo potencial de 5,7 a $6,1 \mathrm{~mm} / \mathrm{d}$ según zonas. Entonces los regantes mantienen una altura de lámina de agua de 15 a $18 \mathrm{~cm}$. Durante la maduración disminuye el consumo (DGA/CEDEX, 2013).

Cultivan también hortalizas y frutales en las tierras más altas de los dos bancos del Ebro y en los bordes occidentales del delta que tienen drenaje natural. En las dos fincas que disponen de sistemas de drenaje subterráneo además del arroz han cultivado hortalizas y actualmente césped para campos deportivos.

\section{Balances de agua}

En la campaña de riego consideran las entradas de agua de lluvia y de riego y las salidas por consumo de agua del arroz y por drenaje superficial de los arrozales. En la de medidas ambientales también la evaporación de la lámina de agua. No contemplan 
filtraciones del Ebro, por estar protegidas las tierras regadas de esas cuencas por las de los dos bancos del río. Tampoco filtraciones de las lagunas interiores, captadas por los desagües de circunvalación y conducidas a las estaciones de evacuación, ni aportes de los ullales. Sin aportes de agua de canal durante episodios sin lluvias significativas que den lugar a percolación puede haber elevación capilar de agua. La diferencia entre las entradas y salidas es el cambio del contenido de agua de cada cuenca $(\Delta W)$, que incluye las variaciones en el nivel de agua en los desagües y las de la lámina de agua en los arrozales. Al estar saturados de agua los campos no hay variación del contenido de humedad en la zona radicular del arroz.

Los balances de los arrozales, expresados en altura de lámina de agua en $\mathrm{mm}$, parten de los caudales $q_{i}$ y de los coeficientes $q_{d r}$, determinados con los caudales $Q$ aforados en las estaciones de salida del agua. Consideran la superficie efectivamente cultivada y regada de arroz en cada cuenca y no su superficie total, porque el drenaje en estas campañas procede mayoritariamente de los retornos de agua de riego, con excepción de algunos cortos períodos con lluvias suficientes para producir escorrentía superficial en los arrozales. También períodos de las series de datos RIADE en los que las diferencias entre los valores máximos y mínimos de $Q$ son más pequeñas. La Tabla 2 resume los resultados de los balances de agua en las campañas de riego de los años 2012-2015.

\begin{tabular}{|c|c|c|c|c|c|}
\hline Fase & $\begin{array}{l}\text { Campaña } \\
\text { de riego }\end{array}$ & $\begin{array}{l}\text { Cuenca } \\
\text { estación }\end{array}$ & $\begin{array}{c}q_{d r} \\
I s^{-1} h^{-1}\end{array}$ & $\begin{array}{c}\Delta W \\
\mathrm{~mm} / \mathrm{d}\end{array}$ & Observaciones \\
\hline \multirow{2}{*}{$\begin{array}{l}\text { Germinación y } \\
\text { emergencia }\end{array}$} & \multirow[t]{2}{*}{2015} & \multirow[t]{2}{*}{ EB7 } & 1,133 & 2,4 & Episodio de 3 días a final de abril \\
\hline & & & 1,642 & $-2,1$ & Episodio de 12 días de mayo \\
\hline \multirow{10}{*}{$\begin{array}{l}\text { Ahijado y } \\
\text { crecimiento }\end{array}$} & \multirow[t]{4}{*}{2013} & EB1 & 0,995 & $<2$ & Episodios varios de 25 días en total \\
\hline & & EB2 & 1,088 & $<2,3$ & $\begin{array}{l}\text { Episodios de } 17 \text { días en total durante las } \\
\text { campañas 2013-2014 }\end{array}$ \\
\hline & & \multirow[t]{2}{*}{ EB8 } & 1,406 & 0,91 & Períodos en las campañas 2013 y 2014 \\
\hline & & & 1,472 & & Episodios con precipitación \\
\hline & \multirow[t]{4}{*}{2014} & EB1 & 0,855 & 3,7 & Episodio de 13 días \\
\hline & & EB2 & $1,7-2,2$ & & Episodios cortos \\
\hline & & EB7 & 1,099 & 1,1 & Episodio de 9 días \\
\hline & & & 1,472 & $-0,1$ & 2 episodios de 28 días con precipitación \\
\hline & \multirow[t]{2}{*}{2015} & \multirow[t]{2}{*}{ EB7 } & 0,655 & 5,8 & Período de 16 días \\
\hline & & & 1,267 & 0,2 & Período de 17 días \\
\hline \multirow{6}{*}{$\begin{array}{l}\text { Espigado y } \\
\text { formación del } \\
\text { grano }\end{array}$} & \multirow[t]{3}{*}{2013} & EB1 & 0,783 & $<4$ & $\begin{array}{l}\text { Varios episodios de } 28 \text { días durante las } \\
\text { campañas 2012-2014 }\end{array}$ \\
\hline & & EB2 & 1,127 & $<1$ & Periodo de 9 días \\
\hline & & EB8 & 1,951 & & Período de 24 días \\
\hline & \multirow[t]{3}{*}{2014} & \multirow[t]{2}{*}{ EB7 } & 0,804 & 4,4 & 2 episodios de 16 días \\
\hline & & & 2,272 & $-5,3$ & 9 días con lluvias \\
\hline & & EB8 & 0,733 & & Período de 28 días \\
\hline \multirow[t]{11}{*}{$\begin{array}{l}\text { Maduración } \\
\text { del grano }\end{array}$} & \multirow[t]{2}{*}{2012} & \multirow[t]{2}{*}{ EB1 } & 1,579 & & $\begin{array}{l}\text { Período de } 29 \text { días en septiembre durante las } \\
\text { campañas 2012-2013 }\end{array}$ \\
\hline & & & 0,865 & & Episodio de 6 días en octubre \\
\hline & \multirow[t]{3}{*}{2013} & \multirow[t]{3}{*}{ EB8 } & 1,914 & -4 & 11 días en agosto-septiembre \\
\hline & & & 1,407 & & 15 días en la $2^{a}$ decena de septiembre \\
\hline & & & 0,819 & & 16 días en septiembre-octubre \\
\hline & \multirow[t]{6}{*}{2014} & \multirow[t]{2}{*}{ EB2 } & 2,800 & & Período de 12 días en agosto-septiembre \\
\hline & & & 2,400 & $-11,1$ & Período de la $2^{\mathrm{a}}$ decena de septiembre \\
\hline & & \multirow[t]{3}{*}{ EB7 } & 2,155 & $-7,5$ & Episodios de 23 días en agosto-septiembre \\
\hline & & & 1,325 & & 3 días en la $4^{\mathrm{a}}$ semana de septiembre \\
\hline & & & 1,199 & & 6 días en la $1^{\mathrm{a}}$ semana de octubre \\
\hline & & EB8 & 0,865 & & En agosto-septiembre \\
\hline
\end{tabular}

Tabla 2. Resultados de los balances de agua durante campañas de riego (CHE-CEDEX, 2016) 
La lámina de agua en los arrozales aumenta al comienzo de la campaña y disminuye al final. También hay variaciones durante períodos cortos de unos 5 días por aplicación de tratamientos. Durante períodos más estables de los niveles de agua en desagües y arrozales, las diferencias determinadas podrían deberse a que el consumo real del arroz es mayor que la $E T_{c}$ calculada, a que $q_{i}$ es menor que el nominal supuesto y a errores propios de las estimaciones de las cantidades de drenaje a partir de los aforos RIADE y de las superficies cultivadas de arroz. Los resultados indican que durante las dos fases del cultivo en las que la lámina de agua es más constante, la cantidad de agua consumida por el arroz es similar a la evacuada por drenaje para el control de la temperatura y de la salinidad de la lámina de agua de los arrozales.

En las campañas de medidas ambientales los balances consideran los caudales de agua de canal y las precipitaciones diarias. A falta de datos sobre la evaporación del agua $(E)$ de la lámina de los campos inundados toman la $E T_{0}$. La superficie de los campos inundados coincide con la de los arrozales en los años estudiados. Los caudales $q_{d r}$ durante las medidas ambientales varían en función de las precipitaciones, más probables en otoño que durante la campaña de riego. Esos caudales han sido menores en octubre mientras los campos vuelven a llenarse de agua tras la recolección del arroz. La Tabla 3 incluye los resultados durante los años 2012-2015 en las 4 cuencas estudiadas.

\begin{tabular}{|c|c|c|c|c|c|}
\hline Mes & $\begin{array}{c}\text { Campaña } \\
\text { de } \\
\text { medidas }\end{array}$ & $\begin{array}{l}\text { Cuenca } \\
\text { estación }\end{array}$ & $\begin{array}{l}q_{d r} \\
\text { Is }^{-1} \mathrm{ha}^{-1}\end{array}$ & $\begin{array}{c}\Delta W \\
\mathrm{~mm} / \mathrm{d}\end{array}$ & Observaciones \\
\hline \multirow[t]{4}{*}{ Octubre } & \multirow[t]{2}{*}{2013} & EB1 & 1,188 & $-0,8$ & Periodo de 9 días \\
\hline & & EB8 & 1,005 & 2,1 & Periodo de 15 días \\
\hline & \multirow[t]{2}{*}{2014} & EB7 & 1,760 & $-4,3$ & Periodo de 22 días con lluvias \\
\hline & & EB8 & 0,655 & 5,4 & $\begin{array}{l}2 \text { periodos de } 27 \text { días en total; el segundo hasta el } 4 \\
\text { de noviembre }\end{array}$ \\
\hline \multirow[t]{3}{*}{ Noviembre } & \multirow[t]{3}{*}{2014} & EB2 & 2,046 & $-7,0$ & 3 episodios de 8 días en total con lluvias previas \\
\hline & & EB7 & 1,988 & $-5,8$ & Periodo de 9 días con lluvia \\
\hline & & EB8 & 0,653 & 5,8 & 3 períodos de 19 días en total \\
\hline \multirow[t]{5}{*}{ Diciembre } & 2012 & EB1 & 0,986 & 2,0 & Episodio de 3 días durante la $1^{\mathrm{a}}$ semana \\
\hline & \multirow[t]{4}{*}{2014} & \multirow[t]{3}{*}{ EB2 } & 3,979 & $-16,3$ & Episodios con lluvias previas en noviembre-diciembre \\
\hline & & & 1,453 & $-2,2$ & Episodio de 6 días en la $2^{\mathrm{a}}$ semana \\
\hline & & & 2,297 & $-6,8$ & Episodio de 6 días en la $3^{\mathrm{a}}$ semana con lluvias previas \\
\hline & & EB8 & 0,070 & 11,0 & Período de 17 días hasta el 3 de enero de 2015 \\
\hline \multirow[t]{3}{*}{ Enero } & 2013 & EB1 & 1,633 & $-4,2$ & $2^{\mathrm{a}}$ quincena de diciembre de 2012 y enero de 2013 \\
\hline & 2014 & EB2 & 1,352 & $-3,0$ & A final de mes \\
\hline & 2015 & EB8 & 0,095 & 10,7 & Período de 11 días \\
\hline
\end{tabular}

Tabla 3. Resultados de los balances de agua durante campañas de medidas ambientales

(CHE-CEDEX, 2016)

Sin lluvias de octubre a diciembre, aproximadamente el 80 por ciento del agua del canal aportada a los campos anegados es evacuada por los sistemas de drenaje superficial. Episodios de lluvias en otoño aumentan significativamente el caudal $q_{d r}$ que supera al $q_{i}$. En enero aún sin lluvia, el caudal $q_{d r}$ es mayor que $q_{i}$ debido al desagüe progresivo de los campos al final de la campaña de medidas ambientales.

La Tabla 4 muestra los resultados en el período sin riego, entre primeros de febrero y mediados de abril, durante los años 2013-2015. Al inicio de este período, los caudales $q_{d r}$ se debieron al desagüe progresivo de los campos inundados hasta final de enero. Después de la segunda decena de febrero, esos caudales dependieron fundamentalmente de la escorrentía superficial del exceso de agua de lluvia. Pudo haber también una pequeña contribución del drenaje subterráneo hacia la red de desagües y de los retornos del riego de las huertas de los bancos que se riegan con agua del Ebro, así como de las situadas en las periferias occidentales de ambos hemideltas.

Pequeñas precipitaciones originan inmediatamente aumentos de los caudales de drenaje. Este hecho indica que el contenido de humedad de los campos tras las medidas ambientales es alto, próximo a capacidad de campo. Los suelos solamente pierden humedad por efecto de la $E$ de su superficie que en esa época (final del invierno y comienzo 
de la primavera) está libre de vegetación. El aumento del caudal $q_{d r}$ es inmediato al período con lluvias.

\begin{tabular}{|c|c|c|c|c|c|}
\hline Mes & $\begin{array}{c}\text { Campaña } \\
\text { de } \\
\text { medidas }\end{array}$ & $\begin{array}{l}\text { Cuenca } \\
\text { estación }\end{array}$ & $\begin{array}{c}q_{d r} \\
\mathrm{Is}^{-1} \mathrm{ha}^{-1}\end{array}$ & $\begin{array}{c}\Delta W \\
\mathrm{~mm} / \mathrm{d}\end{array}$ & Observaciones \\
\hline \multirow[t]{8}{*}{ Febrero } & \multirow[t]{2}{*}{2013} & \multirow[t]{2}{*}{ EB1 } & 0,119 & $-2,5$ & $1^{\mathrm{a}}$ y $2^{\mathrm{a}}$ decena de febrero \\
\hline & & & 0,223 & $-2,8$ & $4^{\mathrm{a}}$ semana de febrero con lluvia \\
\hline & \multirow[t]{4}{*}{2014} & EB7 & 0,007 & $-1,2$ & Período de 25 días \\
\hline & & EB8 & 0,129 & $-2,7$ & $1^{\mathrm{a}}$ quincena de febrero \\
\hline & & \multirow[t]{2}{*}{ EB2 } & 0,443 & $-4,4$ & $1^{\mathrm{a}}$ decena de febrero \\
\hline & & & 0,167 & $-2,4$ & $2^{\mathrm{a}}$ decena de febrero \\
\hline & \multirow[t]{2}{*}{2015} & EB7 & 0,051 & $-2,4$ & $2^{\mathrm{a}}$ quincena de febrero \\
\hline & & EB8 & 0,016 & $-2,4$ & $2^{\mathrm{a}}$ quincena de febrero y $1^{\mathrm{a}}$ quincena de marzo \\
\hline \multirow[t]{5}{*}{ Marzo } & 2013 & EB1 & 0,372 & $-5,4$ & $2^{\mathrm{a}}$ semana de marzo con precipitación previa \\
\hline & \multirow[t]{3}{*}{2014} & EB7 & 0,040 & $-4,0$ & Período de 17 días \\
\hline & & EB8 & 0,050 & $-2,8$ & Período de 25 días \\
\hline & & EB1 & 0,278 & $-4,7$ & Período de 18 días \\
\hline & 2015 & EB8 & 0,031 & 8,7 & Episodio de 12 días con precipitación \\
\hline \multirow[t]{4}{*}{ Abril } & 2013 & EB1 & 0,505 & $-6,5$ & Final de marzo y comienzo de abril \\
\hline & 2014 & EB1 & 0,275 & $-3,8$ & Final de marzo y comienzo de abril \\
\hline & \multirow[t]{2}{*}{2015} & EB7 & 0,010 & $-3,4$ & Período de 16 días \\
\hline & & EB8 & 0,012 & $-3,5$ & $1^{\mathrm{a}}$ quincena de abril \\
\hline
\end{tabular}

Tabla 4. Resultados de los balances de agua durante períodos sin riego (CHE-CEDEX, 2016)

\section{Balances de sales}

Estos balances parten de los de agua teniendo en cuenta las concentraciones de sales respectivas de las entradas y salidas de agua en cada cuenca. Estas concentraciones han sido estimadas a partir de los datos de CE de las aguas, del Ebro en Xerta y de drenaje en las estructuras de su evacuación. Para transformar los datos de CE de RIADE en valores de $S D T$, se han utilizado los coeficientes SDT/CE deducidos de la correlación entre estos parámetros determinada con los resultados de los análisis completos disponibles.

Las entradas de sales en los arrozales de esas cuencas son las que aporta el agua de canal durante las campañas de riego del arroz y de medidas ambientales. También, desde febrero hasta la segunda quincena de abril, las que pudiera aportar el acuífero superficial por elevación capilar de agua durante episodios sin lluvia. No se han considerado aportaciones de sales desde el río Ebro por las razones expuestas anteriormente ni las que aporta la precipitación, porque la concentración de sales en el agua de lluvia es irrelevante comparada con la del agua de riego. Tampoco las aportadas con los fertilizantes por una razón similar. Las salidas de sales de los balances de las cuencas estudiadas incluyen solamente las evacuadas con el agua de drenaje sin tener en cuenta absorción alguna de sales por parte del arroz.

La Figura 2 muestra la variación de los SDT en el agua de un arrozal durante la campaña de riego en las 4 cuencas de drenaje. Como indica algunas diferencias no es posible elaborar un balance medio de sales de las 4 cuencas. Sin embargo, la disminución del contendido de sales de los arrozales determinado en la última fase de cultivo (de 3 a 4 t/ha) es igual o superior a la cantidad de sales aportadas con el agua de riego durante la inundación de los campos y la fase de espigado y formación del grano del arroz, incluso si hay cierto aumento durante la fase de ahijado y crecimiento del arroz. Todo sin contar que durante la campaña de riego puede haber mayor evacuación de sales durante episodios de lluvias que ocasionan mayor cantidad de agua de drenaje superficial. Además, el balance de sales de la cuenca que desagua por la estación EB2 muestra que la cantidad de sales evacuadas es mayor que la de las aportadas con el agua de riego. Esto es un indicio de que en algunas cuencas podría haber eliminación de sales del acuífero superficial. Con el actual riego y drenaje de los arrozales el balance de sales es favorable y no existe riesgo de 
aumento de la salinidad a medio plazo, pero hay que estudiar si el incremento de la salinidad en el espigado y formación del grano del arroz influye en el rendimiento del cultivo.

a)

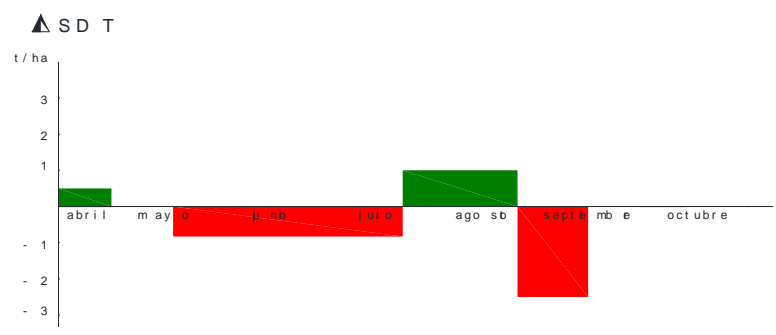

b)
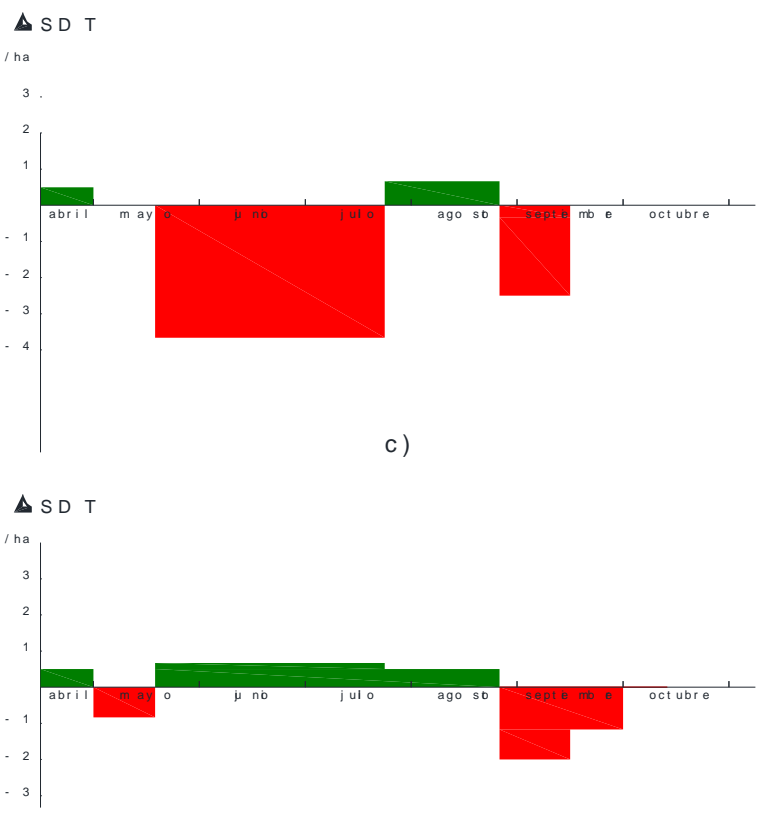

d)

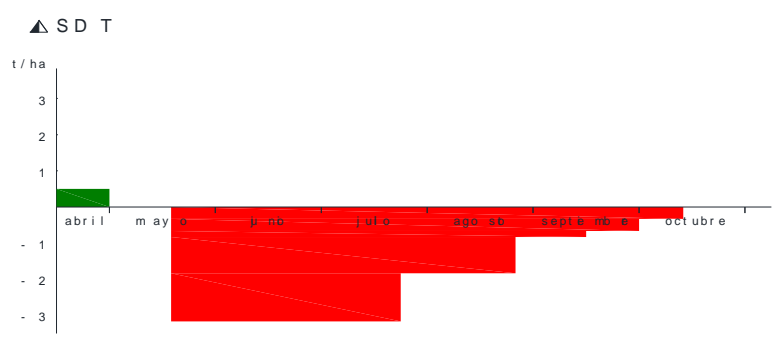

a)

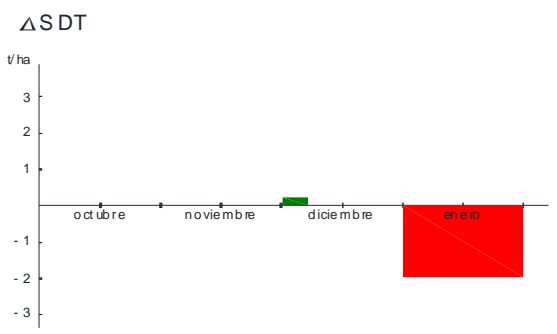

b)

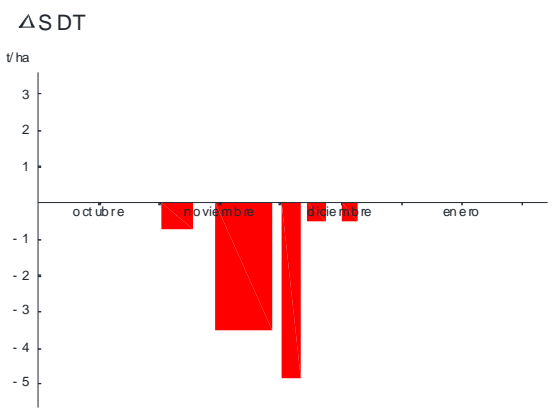

c)

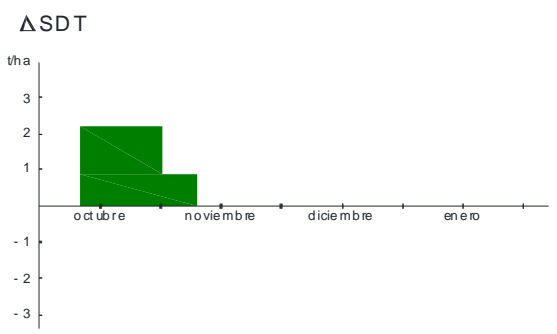

d)

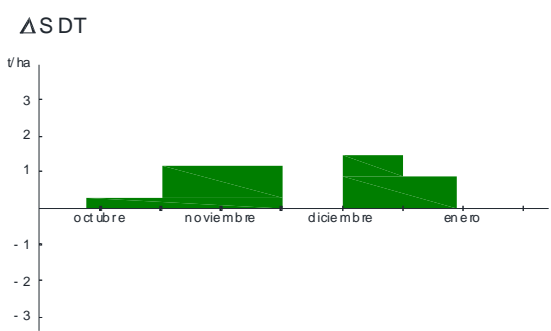

Figura 2. Esquema de la variación de los SDT en el agua de un campo en las cuencas de drenaje durante las campañas de riego y de medidas ambientales (CHE-CEDEX, 2016)

a) EB1; b) EB2; c) EB7; d) EB8

También la Figura 2 muestra la variación de los SDT en el agua de los campos inundados durante las campañas de medidas ambientales. Si se combinan los esquemas de las cuencas de las estaciones EB1, EB2 y EB7 puede obtenerse una idea del balance global durante esta parte del año hidrológico. Los balances están equilibrados porque las entradas y las salidas de sales son muy similares. En ausencia de lluvias aumenta la salinidad en octubre-noviembre al reponerse la lámina de agua de los campos y en 
diciembre-enero disminuye al irse rebajando esa lámina. En el conjunto del período octubreenero, las sales aportadas con el agua del canal son evacuadas a las bahías y al mar.

Son pocos los períodos estudiados de febrero a mitad de abril debido a la escasa coincidencia de datos disponibles de $Q$ y $C E$ del agua de drenaje, pero son suficientes para destacar tres procesos relevantes en lo que se refiere al control de la salinidad: la eliminación de sales con el agua remanente de la inundación de los campos en la campaña de medidas ambientales; la aportación de sales al suelo por elevación capilar de agua freática en períodos sin lluvia; y la eliminación de esas sales con el exceso de agua de lluvia drenada en períodos con precipitación.

\section{Balances de nitratos}

Los balances se basan en los de agua y en la concentración de $\mathrm{NO}_{3}$ del agua de riego y de drenaje. La primera es más constante que la segunda, aunque progresivamente decrece en la campaña de riego para aumentar también paulatinamente durante las medidas ambientales. La segunda disminuye gradualmente a lo largo de la campaña de riego alcanzando valores mínimos en agosto, siendo en ocasiones menor que la del agua de riego. Como un ejemplo, la Tabla 5 muestra los de una hectárea de arrozal en una de las cuencas de drenaje del hemidelta derecho durante dos campañas de riego.

\begin{tabular}{|c|c|c|c|c|c|c|c|c|c|}
\hline \multirow[b]{2}{*}{ Período } & \multirow{2}{*}{$\begin{array}{l}T \\
\mathrm{~d}\end{array}$} & \multicolumn{3}{|c|}{$\begin{array}{c}\text { Nitratos } \\
\text { aportados con el } \\
\text { agua de riego }\end{array}$} & \multicolumn{3}{|c|}{$\begin{array}{c}\text { Nitratos evacuados } \\
\text { con el agua de drenaje }\end{array}$} & \multirow{2}{*}{$\begin{array}{c}\Delta N \\
\mathrm{~kg} / \mathrm{ha}\end{array}$} & \multirow[t]{2}{*}{ Observaciones } \\
\hline & & $\begin{array}{l}\mathrm{NO}_{3 i} \\
\mathrm{mg} / \mathrm{l}\end{array}$ & $\begin{array}{c}\text { I } \\
\mathrm{m}^{3} / \mathrm{ha}\end{array}$ & \begin{tabular}{|c|} 
Nitrato \\
$\mathbf{s}$ \\
$\mathrm{kg} / \mathrm{ha}$
\end{tabular} & $\begin{array}{c}\mathrm{NO}_{3 d r} \\
\mathrm{mg} / \mathrm{l}\end{array}$ & $\begin{array}{c}D r \\
\mathrm{~m}^{3} / \mathrm{ha}\end{array}$ & $\begin{array}{c}\text { Nitratos } \\
\mathrm{kg} / \mathrm{ha}\end{array}$ & & \\
\hline 1 al 15 de mayo & 15 & 11,06 & 2.151 & 23,79 & 7,00 & 618 & 4,33 & 19,46 & $\begin{array}{l}\text { Llenado de arrozales con agua y } \\
\text { fase de plántula del arroz }\end{array}$ \\
\hline 26 al 31 de julio & 6 & 6,67 & 861 & 5,74 & 3,19 & 474 & 1,51 & 4,23 & Crecimiento y ahijado del arroz \\
\hline \begin{tabular}{|l}
11 al 25 de \\
septiembre
\end{tabular} & 15 & 5,90 & 1.788 & 10,55 & 8,29 & 2.215 & 18,36 & $-7,81$ & $\begin{array}{l}\text { Reducción de la lámina de agua } \\
\text { y maduración del arroz }\end{array}$ \\
\hline Totales 2012 & 36 & & 4.800 & 40,08 & & 3.307 & 24,20 & 15,88 & \\
\hline 8 al 15 de mayo & 8 & 8,59 & 1.251 & 10,75 & 8,25 & 885 & 7,30 & 3,44 & $\begin{array}{l}\text { Llenado de arrozales con agua y } \\
\text { fase de plántula del arroz }\end{array}$ \\
\hline 22 mayo-1de junio & 11 & 8,57 & 1.720 & 14,74 & 6,20 & 805 & 4,99 & 9,75 & Crecimiento y ahijado del arroz \\
\hline 20 al 30 de junio & 11 & 7,40 & 1.720 & 12,73 & 6,32 & 873 & 5,52 & 7,21 & Crecimiento y ahijado del arroz \\
\hline 1 al 14 de julio & 14 & 7,99 & 2.189 & 17,49 & 4,40 & 1.192 & 5,24 & 12,25 & Crecimiento y ahijado del arroz \\
\hline 16 al 28 de julio & 13 & 8,50 & 2.033 & 17,28 & 3,19 & 714 & 2,28 & 15,00 & Comienzo del espigado \\
\hline 11 al 22 de agosto & 12 & 7,93 & 1.877 & 14,88 & 2,23 & 680 & 1,52 & 13,37 & Espigado y formación de grano \\
\hline Totales 2013 & 69 & & 10.790 & 87,87 & & 5.149 & 26,85 & 61,02 & Totales \\
\hline $\begin{array}{l}5 \text { al } 15 \text { de } \\
\text { septiembre, } 2013\end{array}$ & 11 & 8,84 & 1.549 & 13,69 & - & 1.741 & - & - & $\begin{array}{l}\text { Vaciado de agua y maduración } \\
\text { del arroz }\end{array}$ \\
\hline
\end{tabular}

Tabla 5. Balances de nitratos en una hectárea de arrozal de la cuenca de drenaje EB1 durante las campañas de riego de 2012 y 2013 (DGA/CEDEX, 2013)

El contenido de $\mathrm{NO}_{3}$ de la lámina del arrozal aumenta al llenarlos de agua. A lo largo de la campaña de riego buena parte de los $\mathrm{NO}_{3}$ aportados con el agua de riego es consumida por el arroz, progresivamente del 50 al 85 por ciento durante el crecimiento y ahijado y hasta el 90 por ciento en el espigado y formación de grano. Durante el desagüe de los arrozales para la recolección, parte de los $\mathrm{NO}_{3}$ acumulados son evacuados con el agua de drenaje, pero en menor cuantía que los aportados al inicio de la campaña.

Durante la campaña de medidas ambientales del año hidrológico 2012/2013, la concentración del agua del canal fue mayor que durante la de riego. La Tabla 6 muestra los balances de $\mathrm{NO}_{3}$ en una hectárea de campo inundado en la cuenca de drenaje EB1. 


\begin{tabular}{|c|c|c|c|c|c|c|c|c|}
\hline \multirow[b]{2}{*}{ Período } & \multirow[b]{2}{*}{$\begin{array}{l}T \\
\mathrm{~d}\end{array}$} & \multicolumn{3}{|c|}{$\begin{array}{l}\text { Nitratos aportados } \\
\text { con el agua de riego }\end{array}$} & \multicolumn{3}{|c|}{$\begin{array}{l}\text { Nitratos evacuados con } \\
\text { el agua de drenaje }\end{array}$} & \multirow[b]{2}{*}{$\begin{array}{c}\Delta N \\
\mathrm{~kg} / \mathrm{ha}\end{array}$} \\
\hline & & $\begin{array}{l}\mathrm{NO}_{3 i} \\
\mathrm{mg} / \mathrm{l}\end{array}$ & \begin{tabular}{|c|}
$I$ \\
$\mathrm{~m}^{3} / \mathrm{ha}$
\end{tabular} & $\begin{array}{c}\text { Nitratos } \\
\mathrm{kg} / \mathrm{ha}\end{array}$ & $\begin{array}{c}\mathrm{NO}_{3 d r} \\
\mathrm{mg} / \mathrm{l}\end{array}$ & $\begin{array}{c}D r \\
\mathrm{~m}^{3} / \mathrm{ha}\end{array}$ & $\begin{array}{c}\text { Nitratos } \\
\mathrm{kg} / \mathrm{ha}\end{array}$ & \\
\hline $\begin{array}{l}25 \text { de noviembre a } 7 \\
\text { de diciembre, } 2012 \\
\end{array}$ & 13 & 13,95 & 1.651 & 23,03 & 29,63 & 411 & 12,18 & 10,85 \\
\hline $\begin{array}{l}8 \text { al } 13 \text { de diciembre, } \\
2012\end{array}$ & 6 & 14,06 & 762 & 10,71 & 25,18 & 447 & 11,26 & $-0,54$ \\
\hline $\begin{array}{l}14 \text { al } 31 \text { de diciembre, } \\
2012\end{array}$ & 18 & 13,39 & 2.286 & 30,61 & 19,55 & 2.460 & 48,09 & $-17,48$ \\
\hline 1 al 18 de enero, 2013 & 18 & 12,53 & 2.286 & 28,64 & 8,37 & 2.102 & 17,59 & 11,05 \\
\hline Totales & 55 & & 6.985 & 93,00 & & 5.420 & 89,12 & 3,88 \\
\hline
\end{tabular}

Tabla 6. Balances de nitratos en un campo inundado de la cuenca de drenaje EB1 durante las medidas ambientales del año 2012/2013 (DGA/CEDEX, 2013)

Las cifras de entradas y salidas de $\mathrm{NO}_{3}$ son similares. El campo no retiene $\mathrm{NO}_{3}$ porque no hay cultivo que los consuma.

\section{Efectos del riego de los arrozales sobre la calidad del agua}

La masa de sales y $\mathrm{NO}_{3}$ evacuadas al Ebro y a su brazo de Migjorn es mucho menor que la aportada al mar y a las bahías, porque el área que drena a esas masas de agua también lo es. La entrada de agua de drenaje con sales y $\mathrm{NO}_{3}$ a las lagunas es puntual y limitada a excesos de agua de lluvia con concentraciones bajas.

La masa aportada a las bahías y al mar no se puede cuantificar con precisión en su totalidad, por carecer de datos de $Q, S T D$ y $\mathrm{NO}_{3}$ en el agua de drenaje de todas las cuencas que vierten a esas masas. Sin embargo, adoptando valores medios de los $q_{d r}$ determinados en los balances de agua durante la campaña de riego puede estimarse que durante el crecimiento, ahijado y espigado hasta el inicio de la maduración del arroz, las cuencas con datos evacuaron a la bahía de Los Alfaques aproximadamente el 99,4 por ciento de las sales aportadas con el agua de riego. Quedan disueltas en la lámina de agua una pequeña cantidad de sales que todavía tienen los arrozales cuando el arroz ha formado ya su grano (Tabla 7). Asimismo, se vertieron a la bahía durante esas fases del cultivo del arroz el 27,8 por ciento de los $\mathrm{NO}_{3}$ aportados con el agua de riego. Durante la maduración del arroz, en la cuenca EB1 la masa de sales y $\mathrm{NO}_{3}$ evacuada superó a la importada con el agua de riego. Al reducirse la lámina de agua se reduce la masa de sales y $\mathrm{NO}_{3}$ disuelta en el volumen de agua de los arrozales.

Durante la campaña de medidas ambientales en la cuenca EB1 aproximadamente el 83 por ciento de las sales aportadas con el agua del canal fueron evacuadas y un 14 por ciento más de $\mathrm{NO}_{3}$ que los aportados con el agua de riego. El volumen de agua aportado a la bahía durante la campaña de riego, con una salinidad muy inferior a la del agua del mar, explica que la salinidad del agua del estrato superior de la bahía vaya disminuyendo progresivamente a lo largo de la campaña hasta alcanzar en septiembre valores muy inferiores a los de la $C E$ del agua del mar. El efecto sobre el estrato inferior es menor, aunque también hay cierto descenso de la CE del agua al final de la campaña.

Durante las medidas ambientales también las dos cuencas de drenaje aportan a la bahía agua de menor salinidad que el agua de mar, pero las tierras inundadas no retienen los $\mathrm{NO}_{3}$ disueltos en el agua de riego que reciben y son aportados a la bahía.

El riego de los arrozales y la inundación de los campos con agua del canal elevan el nivel freático hasta la superficie del terreno. El acuífero superficial no recibe sales del agua de canal, porque son evacuadas a las masas de agua superficial. Sin embargo, el riego sí produce cierta pérdida de sales del agua de este acuífero elevadas por capilaridad cuando la superficie del suelo se seca en períodos sin riego y en ausencia de lluvias significativas las sales son lavadas por el agua de riego y evacuadas al mar disueltas en el agua de 
drenaje. Tampoco hay aporte de $\mathrm{NO}_{3}$ a ese acuífero porque el arroz absorbe la mayor parte de los aplicados con el agua de riego además de los del abonado.

\begin{tabular}{|c|c|c|c|c|c|c|c|c|c|c|c|c|c|}
\hline \multirow[t]{2}{*}{ Período } & \multirow{2}{*}{$\begin{array}{l}\text { Fase del } \\
\text { arroz }\end{array}$} & \multicolumn{2}{|c|}{ Riego } & \multicolumn{2}{|c|}{$\begin{array}{c}\text { Sales } \\
\text { aportadas }\end{array}$} & \multicolumn{2}{|c|}{$\begin{array}{c}\text { Nitratos } \\
\text { aportados }\end{array}$} & \multicolumn{2}{|c|}{ Drenaje } & \multicolumn{2}{|c|}{$\begin{array}{c}\text { Sales } \\
\text { evacuadas }\end{array}$} & \multicolumn{2}{|c|}{$\begin{array}{c}\text { Nitratos } \\
\text { evacuados }\end{array}$} \\
\hline & & $\mathrm{m}^{3} / \mathrm{ha}$ & $\mathrm{hm}^{3}$ & t/ha & $10^{3} \mathrm{t}$ & kg/ha & $t$ & $\mathrm{~m}^{3} / \mathrm{ha}$ & $\mathrm{hm}^{3}$ & t/ha & $10^{3} \mathrm{t}$ & kg/ha & $t$ \\
\hline \multirow{3}{*}{$\begin{array}{l}1 \text { al } 17 \text { de } \\
\text { mayo } \\
17 \mathrm{~d}\end{array}$} & \multirow{3}{*}{ Plántula } & \multirow{3}{*}{2.556} & 6,43 & \multirow{3}{*}{1,81} & 4,57 & \multirow{3}{*}{26,07} & 65,59 & \multirow{3}{*}{1.087} & 2,73 & 1,46 & 3,66 & 8,08 & 20,32 \\
\hline & & & 7,27 & & 5,16 & & 74,11 & & 3,09 & - & - & - & - \\
\hline & & & 3,95 & & 2,80 & & 40,25 & & 1,68 & - & - & - & - \\
\hline \multirow{3}{*}{$\begin{array}{l}18 \text { mayo al } \\
24 \text { de julio } \\
68 \mathrm{~d}\end{array}$} & \multirow{3}{*}{$\begin{array}{l}\text { Crecimiento } \\
\text { y ahijado }\end{array}$} & \multirow{3}{*}{10.223} & 25,72 & \multirow{3}{*}{5,83} & 14,66 & \multirow{3}{*}{81,37} & 204,74 & 6.345 & 15,96 & 6,28 & 15,80 & 35,15 & 88,44 \\
\hline & & & 29,06 & & 16,57 & & 231,35 & 4.876 & 13,86 & 7,27 & 20,66 & 18,63 & 52,96 \\
\hline & & & 15,78 & & 9,00 & & 125,64 & 4.994 & 7,71 & - & - & - & - \\
\hline \multirow{3}{*}{$\begin{array}{l}25 \text { de julio } \\
\text { al } 25 \text { de } \\
\text { agosto } \\
32 \text { d }\end{array}$} & \multirow{3}{*}{ Espigado } & \multirow{3}{*}{4.811} & 12,10 & \multirow{3}{*}{2,98} & 7,50 & \multirow{3}{*}{38,15} & 95,98 & 1.769 & 4,45 & 1,88 & 4,72 & 5,59 & 14,07 \\
\hline & & & 13,68 & & 8,48 & & 108,46 & 2.488 & 7,07 & 2,02 & 5,73 & 7,86 & 22,35 \\
\hline & & & 7,43 & & 4,61 & & 58,90 & 2.129 & 3,29 & - & - & - & - \\
\hline \multirow{3}{*}{$\begin{array}{l}26 \text { de } \\
\text { agosto al } \\
15 \text { de } \\
\text { septiembre } \\
21 \mathrm{~d}\end{array}$} & \multirow{3}{*}{$\begin{array}{l}\text { Maduración } \\
\text { y desagüe } \\
\text { arrozales }\end{array}$} & \multirow{3}{*}{2.830} & 7,12 & \multirow{3}{*}{1,61} & 4,06 & \multirow{3}{*}{20,21} & 50,85 & \multirow{3}{*}{3.248} & 8,17 & - & - & - & - \\
\hline & & & 8,05 & & 4,59 & & 57,46 & & 9,23 & - & - & - & - \\
\hline & & & 4,37 & & 2,49 & & 31,20 & & 5,01 & - & - & - & - \\
\hline \multirow{3}{*}{$\begin{array}{l}16 \text { al } 30 \text { de } \\
\text { septiembre } \\
15 \mathrm{~d}\end{array}$} & \multirow{3}{*}{$\begin{array}{l}\text { Maduración } \\
\text { y desagüe } \\
\text { arrozales }\end{array}$} & & 3,39 & & 2,78 & & 25,16 & & 5,45 & 4,55 & 11,44 & 17,94 & 45,14 \\
\hline & & 1.348 & 3,83 & 1,11 & 3,14 & 10,00 & 28,43 & 2.164 & 6,15 & - & - & - & - \\
\hline & & & 2,08 & & 1,71 & & 15,44 & & 3,34 & - & - & - & - \\
\hline $\begin{array}{l}153 \text { d de } \\
\text { la } \\
\text { campaña } \\
\text { de riego }\end{array}$ & $\begin{array}{l}153 \mathrm{~d} \text { del } \\
\text { ciclo }(180 \mathrm{~d})\end{array}$ & 21.768 & 150,3 & 13,34 & 92,11 & 175,80 & $1.213,56$ & 14.083 & 97,21 & - & - & - & - \\
\hline $21 \mathrm{de}$ & & & 28,56 & & 27,14 & & 351,91 & & 21,33 & 8,98 & 22,60 & 159,35 & 400,91 \\
\hline 18 de & $\begin{array}{l}\text { Medidas } \\
\text { ambientales }\end{array}$ & 11.353 & 32,28 & 10,79 & 30,66 & 139,87 & 397,65 & 8.476 & 24,10 & - & - & - & - \\
\hline $90 \mathrm{~d}$ & & & 17,53 & & 16,65 & & 215,96 & & 13,09 & - & - & - & - \\
\hline
\end{tabular}

Tabla 7. Volúmenes de agua y masas de sales y nitratos importados con el agua del canal y evacuados a la bahía de Los Alfaques durante las campañas de riego 2012 y 2013 y el período de medidas ambientales 2012/2013 (DGA/CEDEX, 2013)

\section{Conclusiones y recomendaciones}

La salinidad es un problema latente en el delta por ser inherente a su propia condición de llanura de inundación sobre un medio marino. Sin embargo, el riego y drenaje de los arrozales evita la salinización de los suelos y que la mayor parte del delta sea una marisma salobre. Para ello, es necesaria una dotación media anual de agua de unos $23.300 \mathrm{~m}^{3} / \mathrm{ha}$ para satisfacer, casi en partes iguales, el consumo de agua del arroz y controlar la salinidad y temperatura del agua de los arrozales.

Aproximadamente el 80 por ciento del agua de canal aportada a los campos para medidas ambientales en 4 cuencas de drenaje estudiadas es evacuada a las dos bahías, con las sales aportadas con el agua del Ebro e incluso eliminando más sales de los suelos. Períodos sin precipitación ni riego de febrero a abril son más críticos porque entonces el acuífero superficial aporta cierta cantidad de agua por capilaridad y sales a los suelos, pero durante episodios lluviosos pueden evacuarse parte de las sales acumuladas en el suelo en episodios previos sin precipitación.

El aporte de sales del río Ebro a las tierras adyacentes no es relevante, bien por no haber flujo de agua del río al acuífero superficial o por ser la salinidad del río baja en el caso de crecidas, porque entonces la cuña salina se desplaza hacia su desembocadura. 
El arroz consume buena parte de los nitratos proporcionados por el agua de riego, del 50 al 85 por ciento progresivamente en las primeras fases del ciclo del cultivo y hasta el 90 por ciento durante el espigado y maduración del grano. Sin arroz la masa de nitratos aportada al mar por el Ebro sería mayor que la actual.

No es relevante el aporte de agua de drenaje con sales y nitratos disueltos a las lagunas interiores del delta, porque están hidráulicamente aisladas de las tierras regadas por desagües de circunvalación. Solamente entra en esas lagunas agua de exceso de lluvia con baja concentración y agua de canal a petición de los pescadores. Tampoco lo es el aporte al acuífero superficial, más bien el riego y el drenaje eliminan progresivamente sales de este acuífero.

El agua aportada a las dos bahías durante la campaña de riego tiene una salinidad muy inferior a la del agua del mar. Por ello, la salinidad del agua del estrato superior de las bahías disminuye progresivamente a lo largo de la campaña.

Es imprescindible mantener agua dulce sobre el agua salina del acuífero superficial mediante la red de riego, el drenaje superficial de los arrozales, la red de desagües y las compuertas y estaciones de bombeo que evacuan el agua de drenaje, todos ellos actualmente operativos. También el riego de las tierras adyacentes al Ebro para evitar filtraciones de agua del río.

El control de la salinidad de la mayor parte de las tierras del delta obliga actualmente al cultivo permanente del arroz, con una dotación anual de agua más alta que en otros regadíos españoles donde la salinidad no es tan limitante como en el delta. El drenaje subterráneo de las tierras regadas facilita la diversificación de cultivos y un mejor control de la salinidad de los suelos con menos agua y la introducción de otros cultivos de regadío, manteniendo el arroz en la rotación. Esto contribuiría a disminuir las necesidades de agua de riego del delta y facilitaría el control integral de plagas.

\section{Agradecimientos}

A la DGA, a la CHE y al CEDEX por la oportunidad de llevar a cabo los dos estudios de referencia (2013 y 2016) y a las dos comunidades de regantes del delta del Ebro, que con la información proporcionada los hicieron factibles. A la CGRCDE por su invitación a exponer los resultados de estos estudios en sus jornadas técnicas anuales en Amposta.

\section{Referencias bibliográficas}

CHE. 2010. Estudio de la incidencia de la lengua salina de la desembocadura del río Ebro en el acuífero superficial del Delta. Confederación Hidrográfica del Ebro. Zaragoza.

CHE/IGME. 2014. Determinación de parámetros hidrogeológicos en el delta del Ebro. Confederación Hidrográfica del Ebro e Instituto Geológico y Minero de España. Informe técnico provisional. Zaragoza.

CHE/CEDEX. 2016. Estudio de la salinidad de suelos y aguas en las zonas regables del delta del Ebro mediante balances de agua y sales y propuestas de mejora. Confederación Hidrográfica del Ebro. Centro de Estudios y Experimentación de Obras Públicas, Madrid. DGA/CEDEX, 2013. Gestión sostenible del agua de riego en zonas de presiones significativas (detracción/contaminación) sobre masas de agua. Estudio de 3 zonas piloto (85.000 ha). Asistencia técnica, investigación y desarrollo tecnológico en materias de competencia de la Dirección General del Agua. Centro de Estudios y Experimentación de Obras Públicas, Madrid.

INC/Hydrotechnic. 1966. Proyecto de saneamiento y riegos del delta del Ebro. Informe tipo de reconocimiento de la viabilidad técnica y económica del drenaje del delta del Ebro y sustitución del cultivo del arroz por otros cultivos de regadío. Hydrotechnic Corporation S.A. Instituto Nacional de Colonización, Madrid.

IRYDA. 1982. Proyecto de estaciones de bombeo para la mejora del desagüe superficial del delta derecho del Ebro, Tarragona. Instituto Nacional de Reforma y Desarrollo Agrario, Ministerio de Agricultura, Pesca y Alimentación, Madrid. 